\title{
The impact of a visual arts program on quality of life, communication, and well-being of people living with dementia: a mixed-methods longitudinal investigation
}

\author{
Gill Windle, ${ }^{1}$ Karlijn J. Joling, ${ }^{2}$ Teri Howson-Griffiths, ${ }^{3}$ Bob Woods, \\ Catrin Hedd Jones, ${ }^{1}$ Peter M. van de Ven, ${ }^{4}$ Andrew Newman ${ }^{5}$ and Clive Parkinson ${ }^{6}$ \\ ${ }^{1}$ Dementia Services Development Centre, School of Healthcare Sciences, Bangor University, Bangor, Gwynedd, Wales \\ ${ }^{2}$ Department of General Practice and Elderly Care Medicine, Amsterdam Public Health research institute, VU University Medical Center, Amsterdam, \\ the Netherlands \\ ${ }^{3}$ Drama Department, Liverpool Screen School, Liverpool Fohn Moores University, Liverpool, England \\ ${ }^{4}$ Department of Epidemiology and Biostatistics, VU University Medical Center, Amsterdam, the Netherlands \\ ${ }^{5}$ School of Arts and Cultures, Newcastle University, Newcastle-Upon-Tyne, England \\ ${ }^{6}$ Centre of Arts for Health, The Manchester School of Art, Manchester Metropolitan University, Manchester, England
}

ABSTRACT

Background: Research reviews highlight methodological limitations and gaps in the evidence base for the arts in dementia care. In response, we developed a 12-week visual art program and evaluated the impact on people living with dementia through a mixed-methods longitudinal investigation.

Methods: One hundred and twenty-five people living with mild to severe dementia were recruited across three research settings in England and Wales (residential care homes, a county hospital, and community venues). Quantitative and qualitative data on quality of life ( $\mathrm{QoL})$, communication and perceptions of the program were obtained through interviews and self-reports with participants and their carers. Eight domains of wellbeing were measured using a standardized observation tool, and data compared to an alternative activity with no art.

Results: Across all sites, scores for the well-being domains of interest, attention, pleasure, self-esteem, negative affect, and sadness were significantly better in the art program than the alternative condition. Proxy-reported QoL significantly improved between baseline and 3-month follow-up, but no improvements in QoL were reported by the participants with dementia. This was contrasted by their qualitative accounts, which described a stimulating experience important for social connectedness, well-being, and inner-strength. Communication deteriorated between baseline and follow-up in the hospital setting, but improved in the residential care setting.

Conclusions: The findings highlight the potential for creative aging within dementia care, the benefits of art activities and the influence of the environment. We encourage dementia care providers and arts and cultural services to work toward embedding art activities within routine care provision.

Key words: dementia, art, intervention, quality of life, well-being, mixed-methods, longitudinal

\section{Introduction}

There is a growing interest toward including the arts in dementia care for improving outcomes, such as quality of life (QoL) and providing opportunities for social participation. Opportunities to enrich

\footnotetext{
Correspondence should be addressed to: Gill Windle, Dementia Services Development Centre, School of Healthcare Sciences, Bangor University, Ardudwy, Holyhead Road, Bangor, Gwynedd, Wales. Phone: +44 1248 383968. Email: g.windle@bangor.ac.uk. Received 17 Feb 2017; revision requested 17 Jun 2017; revised version received 8 Sep 2017; accepted 18 Sep 2017. First published online 8 November 2017.
}

QoL through creative activities are of international relevance. Despite ongoing pharmacological research, limited definitive medical treatments are currently available for people living with dementia. However, there are gaps in the evidence base relating to the measurable benefits of art activities for people living with dementia (Zeilig et al., 2014). This study contributes new evidence through a longitudinal investigation of the effects of a visual art program for people living with dementia. The program was developed for this research through a theoretical investigation of the 
contextual factors and mechanisms which shape outcomes (Windle et al., 2017). This theoretical basis is recognized as an important first step in the evaluation-implementation process (Medical Research Council, 2006).

\section{The evidence base for the arts in dementia care}

Longitudinal population studies suggest that cultural activities can have a positive impact on health-related outcomes (Gordon-Nesbitt, 2015). Research reviews suggest that art interventions have the potential to improve a broad range of outcomes for people living with dementia, including well-being, QoL, cognitive function, and communication (Mental Health Foundation, 2011; Salisbury et al., 2011; de Medeiros and Basting, 2013; Zeilig et al., 2014; Young et al., 2015). Arts-based activities include dance, music, creative writing, visual art, and singing. There is a growing evidence base for music-based activities (e.g. McDermott et al., 2013), however, despite positive developments, the research reviews highlight methodological limitations for most of the published arts and dementia studies, undermining their potential impact.

The limitations identified in the reviews include the use of small samples of participants that are underpowered for quantitative analysis (Salisbury et al., 2011). In some instances, research designs are poorly described, lacking detail of data collection, and analysis (Young et al., 2015). In others, the content and delivery of the intervention is insufficiently described (Mental Health Foundation, 2011; de Medeiros and Basting, 2013). Others appear to be opportunist samples in very specific contexts, which are useful for early, exploratory qualitative investigation, and reflection, but have limited generalizability (Young et al., 2015). Consequently, despite the existence of considerable practice innovations, the research examining effectiveness and impact has been limited, reducing the potential for evidence-based practice and service improvement.

In relation to the visual arts, helpful findings are emerging from small, exploratory but wellconducted studies of visual arts programs. Camic et al. (2014) applied a mixed-methods pre-post design to an art viewing and art-making program in a gallery setting. Twenty-eight people living with mild to moderate dementia, plus their carers took part in an 8-week program, attending the gallery once a week for two hours. While there were no significant changes in the quantitative outcomes, the qualitative analysis revealed that taking part was an intellectually stimulating learning experience with social interaction, facilitating enjoyment and confidence. There were suggestions of enhanced "cognitive engagement" during the sessions, and some limited reports of the impact carrying over outside the group in terms of new learning and memory enhancement (Camic et al., 2014). Further work (Camic et al., 2015) drew on the 2013 study to suggest that the gallery, open to the public at the same time, enabled an intellectually stimulating learning experience, social interaction, support for carers, and the confidence of the person with dementia, leading to enjoyment, and changed the perceptions of the facilitators and carers in relation to their understanding of dementia.

MacPherson et al. (2009) applied a mixed methods pre-post design, and included behavioral observation to capture "in the moment" engagement in response to an art viewing activity. Fifteen people living with mild to severe dementia visited the National Gallery of Australia for one hour a week over six weeks. The observations indicate that $84.4 \%$ of the participants were "engaged" or "highly engaged" in the activity, regardless of the cognitive impairment. Qualitative findings suggested new learning and discovery of residual abilities, opportunities for social interaction, discussion, and enjoyment. Staff reported that some residents, normally withdrawn, were very animated and talkative on the way back from the gallery. Ullán et al. (2012) undertook an in-depth qualitative exploration of the process and impact of an artviewing and art-making activity in a day-care setting ( $n=21$ people with mild to moderate dementia). They found the participants were interested in learning new things, showing improved confidence in their own skills, and capabilities. There was good recall of the steps in the art-making process and spontaneous conversation.

Although constrained by setting and sample size, such qualitative findings are compelling. They provide insights into the theoretical basis of how and why visual art programs may be effective, and what outcomes may be important, an essential consideration when choosing evaluation tools. They also point strongly to the importance of capturing outcomes that reflect the enrichment of life while living with dementia that are broader than just assessments of cognition.

\section{Aims of this research}

This research aimed to address some of the methodological limitations, build on the exploratory work of MacPherson et al. (2009); Ullán et al. (2012); Camic et al. (2014; 2015), and strengthen the evidence base for visual art programs. Despite the emerging findings in these studies, the methods 
of evaluation still require further development and validation. Visual arts programs are by their nature "complex" in that they contain several interacting components (e.g. settings, the characteristics and responses of those receiving, and delivering programs, etc.), which require careful consideration when designing a study. There is no "gold standard" outcome measure, and the research suggests different approaches may be appropriate. Reflecting guidance for developing and evaluating complex interventions (Medical Research Council, 2006) our research sought to examine different methods of data collection and appropriate outcome measures.

Specifically, we adopted a longitudinal mixedmethods design with repeated measurements taken before, during and after the intervention. We recruited a well-defined target sample and collected quantitative and qualitative data to investigate the following questions:

1. Do well-being, QoL, and communication improve when taking part in a visual art program?

2. Are any effects purely "in the moment" or do they persist over time?

3. How do the participants perceive the impact of taking part?

\section{Methods}

\section{Study design}

A mixed-methods longitudinal design with simultaneous data triangulation undertook four types of data collection. (1) a structured questionnaire including validated outcome measures; (2) qualitative, open-ended questions; (3) self-evaluation of the impact of sessions; (4) behavioral observation of the intervention with an active control condition. The control condition was standardized across the sites as an unstructured social activity with no arts activities. Participants were recruited between May 2014 and May 2015. The peer-reviewed study protocol provides in-depth methodological details, summarized in this paper (Windle et al., $2016 \mathrm{http}: / \mathrm{bmjopen} . \mathrm{bmj} . \mathrm{com} /$ content/bmjopen/6/ 11/e011634.full.pdf).

\section{Setting and participants}

Research site 1 comprised of four residential care facilities in the North East of England. Site 2 was two assessment units within a National Health Service (NHS) county hospital in Derbyshire. Here, the protocol was modified after the second wave of intervention delivery to also include recruitment from a day care service for people with dementia. Site 3 involved three community venues in North Wales (library with a small exhibition area, an arts center with a gallery, an international arts and music venue). Prior to recruitment, the study calculated that a 95\% confidence level with $5 \%$ margin of error and a moderate effect size would require $n=80$. To adjust for attrition, the study sought to over-recruit by at least $25 \%$, resulting in a total required number of 100 participants.

\section{Eligibility criteria}

Participants were included if they had a diagnosis of dementia or evidence of age-related memory impairment and were:

1. A resident in the chosen care home in Newcastle/Tyne and Wear.

2. A resident in the assessment unit/in receipt of services for a minimum of 3 months in Derbyshire.

3. Living in the community in rented/private housing or sheltered housing in North Wales.

Participants were excluded if they had a recent or current episode of major mental illness (other than dementia), were at the end of life or terminally ill, had a debilitating illness that would preclude regular attendance, had a severe uncorrected sensory or communication difficulty and were completely unable to communicate verbally through the medium of either English or Welsh.

\section{Visual arts program}

The development of the program (Windle et al., 2017) identified the theoretical basis and core content, supported by earlier evaluations (Algar, 2012; Gregory and Windle, 2013) and builds on identified good practice. This work was adapted into the working principles of the intervention and standardized as the guidelines for intervention delivery. The program comprises two underpinning factors; dynamic and responsive artistic practice, and a provocative and stimulating aesthetic experience. These are implemented through the content of seven key ingredients for excellent practice: (1) artists understanding dementia; (2) developing a safe and supportive physical and psychological environment for an inspirational visual arts viewing and making program; (3) creating a structure for the viewing and making sessions; (4) delivering sessions that enable inspiration, imagination, creativity, enjoyment, and celebration; (5) developing social connections; (6) personal development; (7) values, ethics, communication, and guiding principles. It involves participative activities with the emphasis on providing a stimulating, high quality experience for the participants, requiring no prior knowledge or skills. It aims to encourage creativity without overwhelming people with complex instructions, be 
interesting and challenging and promote learning where possible. It encompasses meaningful engagement to stimulate imagination, play and discussion, not lectures or the generation of factual exchanges reliant on memory for names and dates. It provides some structure, but created the opportunity for individual expression, fun, and celebrations of achievements in a failure-free environment.

The community arts partners came together for training prior to the start of the study. Artists from each regional organization delivered the sessions in their respective geographical research site (Equal Arts in Newcastle, Nottingham Contemporary in Derbyshire and Denbigh County Council Community Arts in North Wales). A lead artist with prior experience and training in art and dementia facilitated each session, supported by a second artist. Generally, the sessions were structured so that the first half was an art viewing activity, focusing on a small number of artworks, followed by art-making, however, this was flexible and dependent upon the varying degrees of cognitive impairment presented to the artists.

Different materials were provided depending on the art-making task, such as water-based paints, pastels, color pencils, collage material, glue, iPad, quick drying modeling clay, and print-making supplies. Each care home in site 1 had one visit to a local gallery. No gallery visits were made in site 2 due to restrictions on staff leaving the hospital. Where gallery visits were not possible, the artists brought a small selection of artworks to the participants to facilitate discussions. In site 3 , the community libraries had small exhibition areas facilitating art viewing. In the art center, the collection was visited each week.

The research team and arts partners maintained regular contact. Carers and staff were not required to take part although some chose to do so. Each site was expected to deliver four waves of the program, each wave being 12 weeks in length, delivered once a week for two hours to small groups (maximum of 15 participants). A post-intervention review meeting with the artists indicated the program was delivered according to the core principles, and a practitioners' guide, co-produced with the artists, is freely available (Parkinson et al., 2017).

\section{Observed well-being}

The Greater Cincinnati Chapter Well-Being Observation Tool (GCCWBOT) was developed specifically to observe the effects of a visual arts activity on the well-being of people with dementia (Kinney and Rentz, 2005). The original instrument addresses seven domains of well-being with different numbers of indicators (interest, sustained attention, pleasure, negative affect, sadness, selfesteem, and normalcy). Kinney and Rentz (2005) acknowledge the need for further development of the tool, and other studies have adopted it with minor modifications (e.g. Sauer et al., 2014). In this study, we used the tool with five further indicators included; "interest in own work" (interest domain), "negative comments" (self-esteem domain), with an additional domain to capture disengagement represented by "neutral passivity," "staring into space," and "sleeping behaviour."

For each of the eight well-being domains, scores were calculated by counting the number of minutes that the specific behaviors on the indicators were observed in the activities. The indicators "requires verbal prompting or cueing" (attention domain) and "negative self-esteem/comments" (self-esteem domain) were reverse scored for the calculation of the domain scores. As the total observation time for each participant varied, domain scores were standardized by dividing the total domain score by the maximum score that a participant could obtain (i.e. number of items multiplied by minutes observed). These standardized domain scores were then expressed as a percentage (range 0-100), with higher scores indicating a better well-being for the positive domains (interest, attention, pleasure, self-esteem, and normalcy), and a poorer wellbeing for the negative domains (disengagement, sadness, and negative affect). Researchers involved in collecting observation data undertook additional training, followed by independent coding of training videos. Inter-rater agreement was acceptable (intra-class correlation coefficient $>0.70$ ). This was maintained during the course of the study.

\section{Quality of life (QoL)}

QoL was measured with the Dementia Quality of Life (DEMQOL, Smith et al., 2007). The DEMQOL assesses five domains of QoL, including positive and negative emotions, memory, loneliness, and daily activities. It uses both selfreporting (29-items) and ratings by family carer or staff member as proxy (DEMQOL-Proxy; 32items). Higher scores indicate better QoL. It has good internal consistency, inter-rater reliability, and concurrent validity. Respondents are asked to indicate how much they experienced each item during the last week ("a lot" $=1$; "quite a bit" $=2$; "a little" $=3$; "not at all" $=4$ ).

\section{Communication}

Communication and social behavior was assessed with the Holden Communication Scale (HCS; Holden and Woods, 1995), completed by formal 
or informal carers. The scale consists of 12items (range 0-48) covering a range of social behavior and communication variables, including conversation, awareness, pleasure, humor, and responsiveness. Higher scores indicate more difficulty in communication.

\section{Individual perceptions of the intervention from people living with dementia}

For the participants who were able to complete, individual evaluations were sought at the end of sessions via a short, five-item questionnaire developed by the research team. This ascertained individual "in the moment" responses on interest, enjoyment, feelings, friendliness, and sense of achievement. Each question has a visual "smiley faces" response scale, with associated scores ranging from " $1=$ not great" (sad face) through to " $10=$ fantastic" (very happy face). As part of the interview at time 2, perceptions of the impact of taking part in the visual arts program were qualitatively explored.

\section{Demographic and clinical measures}

Demographic data were obtained at baseline on age (years), gender, marital status, ethnicity, education, and socio-economic status (SES) and use of dementia or mood-related medication. The Clinical Dementia Rating (CDR) scale (Hughes et al., 1982) was completed at baseline to classify severity of symptoms. Previous experience of art activity was ascertained.

\section{Procedure}

The study was approved by North Wales research ethics committee - West. All participants' information provided was prepared to be simple, clear, and understandable. Bilingual information (Welsh and English) was prepared in Wales. Researchers met with potential participants and family members or carers to explain the study. If the research considered the potential participant lacked capacity to provide informed consent, a family member or carer was consulted in accordance with the Mental Capacity Act. Qualitative and quantitative data were collected concurrently through an interview at baseline prior to starting the 12-week program, and follow up interviews were conducted three months (Time 2) and six months (Time 3) later. For those who were unable to provide informed consent, their relative/friend/carer was interviewed on their behalf. A proxy response was obtained on behalf of each participant for the DEMQOL-Proxy and the HCS at the same time points.
For the GCCWBOT, data were collected in the comparison condition the week prior to the intervention period (baseline), and to ensure data completeness for all participants, over two intervention sessions for each time-point (Time $1=$ weeks 1 and 2; Time 2 =weeks 11 and 12). One participant was observed at a time, for one minute each. This was followed by a minute for scoring before moving on to observe the next participant for a minute. The self-reported session evaluation forms were completed at the end of these intervention sessions (weeks 1 and 2; weeks 11 and 12).

\section{Quantitative data analysis}

Descriptive statistics and frequency distributions were used to describe the characteristics of the study participants and the self-evaluations. Differences in baseline characteristics between drop-outs and those who completed the 6-month measurement were explored by performing logistic regression analysis. Assumptions of normality were checked by examining the distribution of the residuals of the outcome measures with QQ-plots and histograms.

Differences in change over time for significant improvements in the DEMQOL, the HCS and the normally distributed outcome domains of observed well-being (interest, attention, pleasure, and normalcy) were analyzed with linear mixed-effects models (multi-level modeling). A random effect was included for the correlation between repeated measurements for the same participant. Differences between sites were accounted for by including site as a fixed effect. In addition, models included a fixed effect for time of measurement, the effect of main interest. This was coded as a categorical variable with baseline or control/comparison as the reference category (i.e. time point 1, 2, and 3 ).

For the four outcome domains of the observation tool that were skewed (self-esteem, disengagement, sadness, and negative affect), Generalized Estimating Equations (GEE) models were used with time and site as fixed factors, specified with Tweedie as the distribution and $\log$ as the link function. This distribution has the ability to model skewed data in a flexible way.

Socio-demographic characteristics and level of cognitive impairment were explored as potential confounders by adding them seperately to the models as a covariate to ascertain if they changed the effect estimate by at least $10 \%$ ("changein-estimate method"; Rothman and Greenland, 1998). To assess whether changes in outcomes over time differed between the three sites, we tested interaction effects between the time variable 


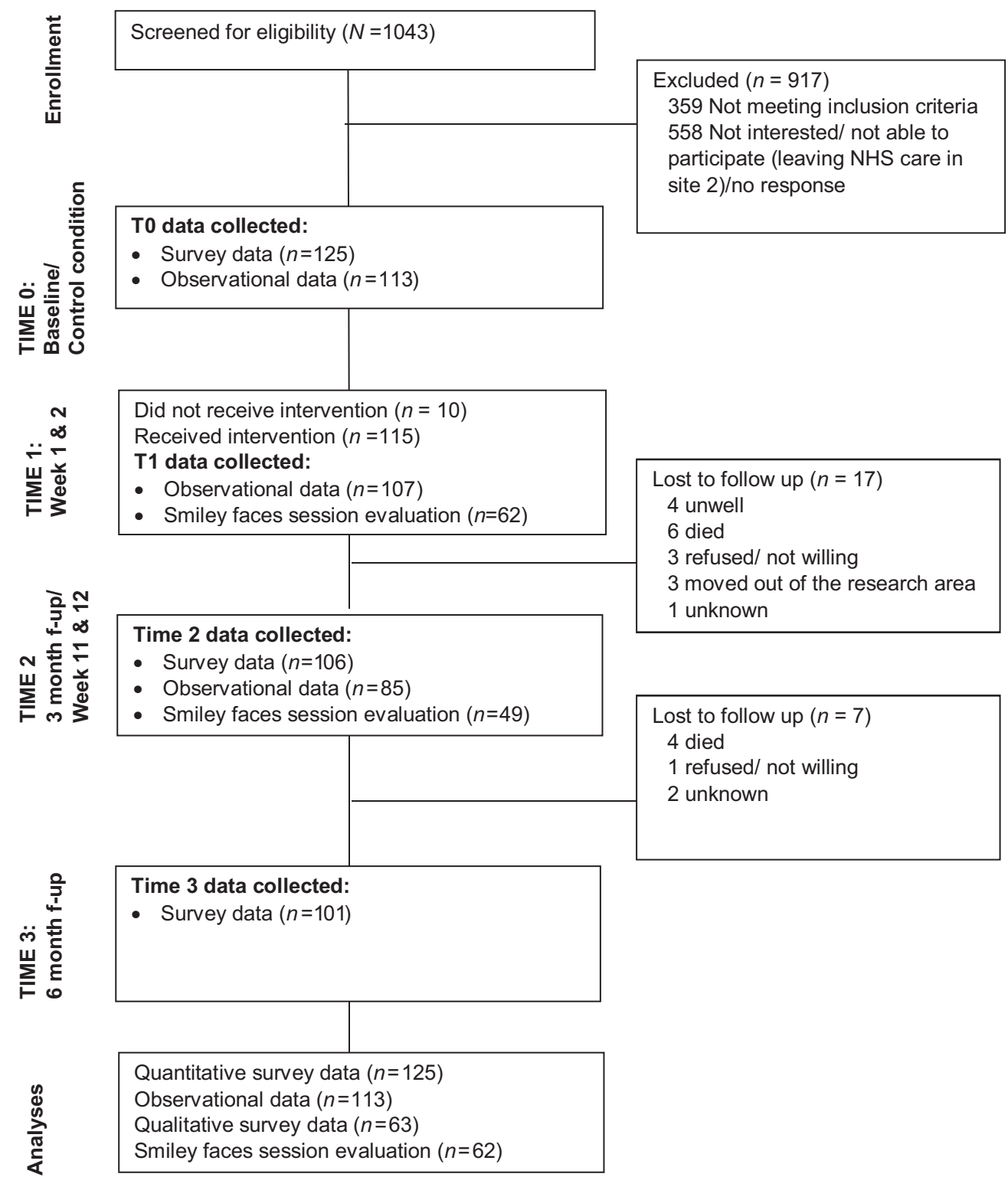

Figure 1. Flow diagram of the study sample. Quantitative and qualitative data were collected in the same interview.

and recruitment site. Statistical significance was considered as two-tailed $p<0.05$. Data were analyzed in SPSS version 22.

\section{Qualitative data analysis}

All responses were fully transcribed and coded in the qualitative software program ATLAS.ti. This was led by $\mathrm{TH}$, who had a minor role in data collection (two interviews). The lead author (GW) had no role in data collection. Utilizing a data driven, inductive thematic analysis (Braun and Clarke, 2006), the themes were determined without applying a pre-conceived coding frame. The coding was an iterative process, evolving through reading, initial coding, and subsequent re-readings of the data. The researcher $(\mathrm{TH})$ developed the initial overarching themes from a table of codes. This was then refined by the two researchers $(\mathrm{TH}$ and GW) through identifying and reviewing sub-themes and supporting data extracts. The interpretation of the data considers the participant's perspectives, sometimes representing conflicts of opinion, and, at other times, shared characteristics.

\section{Results}

\section{Sample characteristics}

Figure 1 presents a flow chart of the recruitment process and data collection. The high numbers excluded after screening are largely reflective of the non-response to recruitment through primary care 
Table 1. Baseline characteristics of the study sample

\begin{tabular}{|c|c|c|c|c|c|c|c|c|}
\hline \multirow[b]{2}{*}{ Age, mean (SD) } & $\begin{array}{r}\quad(\mathrm{CAR} \\
(\Lambda\end{array}$ & $\begin{array}{l}\text { MES) } \\
8)\end{array}$ & \multicolumn{2}{|c|}{$\begin{array}{c}\text { SITE } 2 \\
\text { (NHS HOSPI- } \\
\text { TALWARDS) } \\
(N=23)\end{array}$} & \multicolumn{2}{|c|}{$\begin{array}{c}\text { SITE } 3 \\
(\text { COMMUNITY }) \\
(N=54)\end{array}$} & \multicolumn{2}{|c|}{$\begin{array}{c}\text { TOTAL } \\
\text { SAMPLE } \\
(N=125)\end{array}$} \\
\hline & 84.9 & 7.4 & 81.4 & 4.8 & 78.4 & 9.5 & 81.4 & 8.5 \\
\hline Female gender, $n(\%)$ & 35 & 72.9 & 12 & 52.2 & 26 & 48.1 & 73 & 58.4 \\
\hline \multicolumn{9}{|l|}{ Marital status, $n(\%)$} \\
\hline Married/cohabiting & 10 & 21.3 & 11 & 47.8 & 35 & 64.8 & 56 & 45.1 \\
\hline Single & 3 & 6.4 & 1 & 4.3 & - & - & 4 & 3.2 \\
\hline Widowed & 30 & 63.8 & 11 & 47.8 & 11 & 20.4 & 52 & 42.9 \\
\hline Divorced/separated & 4 & 8.5 & - & - & 8 & 14.8 & 12 & 9.7 \\
\hline \multicolumn{9}{|l|}{ Ethnicity, $n(\%)$} \\
\hline White & 44 & 95.7 & 22 & 95.7 & 53 & 98.1 & 119 & 97.5 \\
\hline Asian/Asian British & - & - & - & - & 1 & 1.9 & 1 & 0.8 \\
\hline Other & 2 & 4.2 & - & - & - & - & 2 & 1.6 \\
\hline $\begin{array}{l}\text { Age leaving FT } \\
\text { education, mean } \\
\text { (SD) }\end{array}$ & 15.8 & 2.7 & 15.1 & 1.7 & 16.2 & 2.4 & 15.9 & 2.5 \\
\hline \multicolumn{9}{|l|}{ Educational level, $n(\%)$} \\
\hline Low & 23 & 47.9 & 17 & 73.9 & 31 & 57.4 & 71 & 64.0 \\
\hline Middle & 4 & 8.3 & - & - & 9 & 16.7 & 13 & 11.7 \\
\hline High & 8 & 16.7 & 5 & 21.7 & 14 & 25.9 & 27 & 24.3 \\
\hline \multicolumn{9}{|c|}{ Level of main activity/ occupation (SES), $n(\%)$} \\
\hline Unskilled & 3 & 6.3 & 4 & 17.4 & 1 & 1.9 & 8 & 7.6 \\
\hline Partly skilled & 3 & 6.3 & 5 & 21.7 & 13 & 24.1 & 21 & 20.0 \\
\hline Skilled (manual) & 16 & 33.3 & 2 & 8.7 & 6 & 11.1 & 24 & 22.9 \\
\hline $\begin{array}{l}\text { Skilled } \\
\text { (non-manual) }\end{array}$ & 9 & 18.8 & - & - & 6 & 11.1 & 15 & 14.3 \\
\hline $\begin{array}{l}\text { Managerial/ } \\
\text { Technical }\end{array}$ & & & 3 & 13.0 & 16 & 29.6 & 19 & 18.1 \\
\hline Professional & 9 & 18.8 & 3 & 13.0 & 6 & 11.1 & 18 & 17.1 \\
\hline $\begin{array}{l}\text { Level of main } \\
\text { activity/occupation } \\
\text { (SES, 0-5), mean } \\
\text { (SD) }\end{array}$ & 2.7 & 1.5 & 2.1 & 1.9 & 2.9 & 1.5 & 2.7 & 1.6 \\
\hline \multicolumn{9}{|l|}{ CDR rating, $n(\%)$} \\
\hline 0.5 Questionable & 6 & 12.5 & 4 & 17.4 & 25 & 46.3 & 35 & 28.2 \\
\hline 1 Mild & 18 & 37.5 & 10 & 43.5 & 27 & 50.0 & 55 & 44.4 \\
\hline 2 Moderate & 8 & 16.7 & 6 & 26.1 & 2 & 3.7 & 16 & 12.9 \\
\hline 3 Severe & 16 & 33.3 & 2 & 8.7 & - & - & 18 & 14.5 \\
\hline \multicolumn{9}{|c|}{ Use of dementia or mood related medication, $n(\%)$} \\
\hline None & 31 & 67.4 & 10 & 43.5 & 15 & 27.8 & 56 & 45.5 \\
\hline $\begin{array}{l}\text { Only dementia } \\
\text { related }\end{array}$ & 11 & 23.9 & 5 & 21.7 & 27 & 50.0 & 43 & 35.0 \\
\hline Only mood related & 4 & 8.7 & 4 & 17.4 & 3 & 5.6 & 11 & 8.9 \\
\hline Both & 0 & - & 4 & 17.4 & 9 & 16.7 & 13 & 10.6 \\
\hline
\end{tabular}

in site 3. One hundred and twenty five people met all inclusion criteria and consented to take part in the study.

Table 1 presents the baseline socio-demographic and clinical characteristics. As expected, those from the residential care homes (site 1) were older, more often female, living alone, and widowed or divorced/separated. Those in the community (North Wales) had less severe cognitive impairements and used more often mood or dementia-related medication. Just over one-third $(37 \%)$ had not previously participated in visual arts activities. Across sites, the participants attended an average of seven sessions $(S D=3.83)$. Ten people $(8 \%)$ did not attend any of the sessions after providing baseline data. 


\section{Attrition analysis}

Long term follow-up data at six months after enrolment were collected from 101 participants $(80.8 \%)$. No differences in baseline characteristics were found between participants who provided data at all collection time-points, and those who dropped-out. Also, there were no differences in dropout between the three sites.

\section{Changes in outcomes over time}

WELL-BEING

Table 2 shows the mean scores and changes over time adjusted for confounding variables. The domain "interest" significantly improved and "negative affect" significantly decreased during both intervention time-points compared with the baseline condition. Compared to the baseline social activity, a significant improvement was observed during the intervention at time 1 (weeks 1 and 2) for the outcome domains "attention" and "pleasure," and a significant reduction between baseline activity and the intervention at time 1 was observed for the domain of "sadness." Furthermore, a significant improvement on "selfesteem" was found at time 2 (weeks 11 and 12) compared to the baseline activity. There were no changes over time for the domains "normalcy" and "disengagement" (Table 2). The moderation analyses found no differences between sites in changes over time on any of the well-being domains.

\section{QUALITY OF LIFE (DEMQOL)}

Table 3 shows the unadjusted mean scores and the changes over time adjusted for SES for both DEMQOL measures. No significant change in the DEMQOL was found over time. According to the DEMQOL Proxy, the QoL of those with dementia improved significantly at time 2 compared to baseline, but this improvement was not sustained at time 3. No significant differences between the sites were found on the DEMQOL and DEMQOL Proxy (see supplementary file 1, available as supplementary material attached to the electronic version of this paper at https://doi.org/10.1017/ S1041610217002162)).

\section{COMMUNICATION AND SOCIAL BEHAVIOR}

Adjusting for age, marital status, and level of dementia, Table 3 shows that scores on the HCS increased significantly between baseline $(M=16.0)$ and time $3(M=17.5)$, indicating a worsening in communication and social behavior in the person with dementia, although there was no change from baseline to time 2 (Table 3 ). We found a significant interaction with site $(\mathrm{F}(4,197.56)=$ $4.97 p=0.001)$. The stratified analyses showed that there was no change in communication for site 3 (community) participants. Site 2 (NHS) participants deteriorated between baseline and time $2(\beta=7.49, t=3.62, p=0.001,95 \%$ CI $3.21-$ $11.75)$ and time $3(\beta=5.63, t=3.38, p=0.003$, 95\% CI 2.17-9.08). In contrast site 1 (care home) participants improved between baseline and time 2 $(\beta=-2.12, t=-2.01, p=0.0,95 \%$ CI -4.23 to $-0.02)$, although this was not sustained at time 3 .

INDIVIDUAL PERCEPTIONS OF THE VISUAL

\section{ARTS INTERVENTION}

Sixty-three people living with dementia discussed the impact of the program at the followup interview, with five themes reflecting their experiences of taking part (Reflection, opinion and recall of activities; well-being and inner strength; social connectedness; stimulating experience; factors influencing participation. See supplementary file 2). Most participants responded positively. In some cases, the art focus provided the main incentive to attend, for others the social aspect was more important, and a number perceived the benefits of both, making connections between the art and social aspects. The program content was a stimulating experience, being a subject for conversation and learning more about other participants through discussion and artmaking. Articulating the unique impact of the art activity was more challenging. Nevertheless, most respondents were able to express a sense of enjoyment and pleasure in taking part in the art program, improving their well-being (such as improving their mood or emotional state), or, were able to describe and reflect on particular techniques they had most appreciated in the art group.

Participants also identified that even if they did not perceive their own work as aesthetically pleasing, they conveyed pleasure in the process of engaging with making art, and many expressed pride in their sense of achievement.

This expression of opinions suggests a good recall of the activities. Some key factors influencing participation (accessing transport, the venue, poor health, and stigma) are important considerations for future services regarding equity of access (see File S2). The evaluations provided at the end of the sessions indicate that the participants found the sessions interesting, enjoyable, and friendly that they felt involved and had achieved something (see Figure 2).

\section{Discussion}

An arts program developed for the purpose of this research was examined simultaneously in three settings with people ranging from mild to severe dementia. As far as we are aware, this is the largest 
Table 2. longitudinal changes for domains of observed well-being

\begin{tabular}{|c|c|c|c|c|c|c|c|c|c|c|c|c|c|c|c|c|c|}
\hline \multirow{2}{*}{$\begin{array}{l}\text { LINEAR MIXED } \\
\text { MODELS }\end{array}$} & \multicolumn{3}{|c|}{$\begin{array}{c}\text { SITE } 1 \\
\text { CARE HOMES }\end{array}$} & \multicolumn{3}{|c|}{$\begin{array}{c}\text { SITE } 2 \\
\text { NHS UNITS }\end{array}$} & \multicolumn{3}{|c|}{$\begin{array}{c}\text { SITE } 3 \\
\text { COMMUNITY }\end{array}$} & \multicolumn{3}{|c|}{ TOTAL SAMPLE } & \multicolumn{5}{|c|}{ TIME } \\
\hline & $N$ & MEAN & $(\mathrm{SD})$ & $N$ & MEAN & $(\mathrm{SD})$ & $N$ & MEAN & $(\mathrm{SD})$ & I & MEAN & $(\mathrm{SD})$ & $\mathrm{B}(\mathrm{TIME})^{*}$ & $95 \% \mathrm{CI}$ & $\mathrm{T}$ & $\mathrm{DF}$ & $\mathrm{p}$ \\
\hline \multicolumn{18}{|l|}{ Interest } \\
\hline Baseline - control & 46 & 25.5 & 21.3 & 18 & 52.5 & 28.9 & 49 & 22.0 & 9.1 & 85 & 28.3 & 21.4 & Ref & & & & \\
\hline Time 1 & 44 & 42.8 & 23.2 & 20 & 50.5 & 18.8 & 43 & 33.0 & 9.5 & 113 & 40.3 & 19.0 & 11.8 & $(7.14 ; 16.53)$ & 5.0 & 165.3 & $<0.001$ \\
\hline Time 2 & 35 & 40.3 & 22.5 & 12 & 47.9 & 18.3 & 38 & 33.4 & 6.7 & 107 & 38.3 & 17.2 & 10.9 & $(5.93 ; 15.96)$ & 4.3 & 172.9 & $<0.001$ \\
\hline \multicolumn{18}{|l|}{ Attention } \\
\hline Baseline - control & 46 & 46.9 & 28.8 & 18 & 67.5 & 21.0 & 49 & 59.4 & 18.2 & 85 & 55.6 & 24.5 & Ref & & & & \\
\hline Time 1 & 44 & 59.7 & 26.1 & 20 & 71.9 & 16.5 & 43 & 63.1 & 15.2 & 113 & 63.3 & 20.9 & 5.8 & $(0.53 ; 11.07)$ & 2.2 & 158.2 & 0.031 \\
\hline Time 2 & 35 & 52.8 & 33.9 & 12 & 69.5 & 20.4 & 38 & 67.1 & 14.6 & 107 & 61.5 & 25.9 & 5.2 & $-(0.48 ; 10.82)$ & 1.8 & 163.4 & 0.073 \\
\hline \multicolumn{18}{|l|}{ Pleasure } \\
\hline Baseline - control & 46 & 26.0 & 26.8 & 18 & 26.0 & 22.2 & 49 & 17.9 & 17.1 & 85 & 22.5 & 22.5 & Ref & & & & \\
\hline Time 1 & 44 & 33.2 & 27.8 & 20 & 25.9 & 17.0 & 43 & 20.4 & 14.4 & 113 & 26.7 & 22.0 & 6.2 & $(0.82 ; 11.66)$ & 2.3 & 162.5 & 0.024 \\
\hline Time 2 & 35 & 35.6 & 34.3 & 12 & 25.5 & 18.9 & 38 & 15.5 & 11.2 & 107 & 25.2 & 25.8 & 1.7 & $(-4.06 ; 7.56)$ & 0.6 & 168.6 & 0.553 \\
\hline \multicolumn{18}{|l|}{ Normalcy } \\
\hline Baseline - control & 46 & 22.9 & 27.4 & 18 & 46.1 & 20.2 & 49 & 27.8 & 10.5 & 85 & 28.7 & 21.8 & Ref & & & & \\
\hline Time 1 & 44 & 21.4 & 26.9 & 20 & 41.5 & 15.3 & 43 & 26.4 & 9.4 & 113 & 27.2 & 20.6 & -1.5 & $(-6.08 ; 3.04)$ & -0.7 & 157.5 & 0.512 \\
\hline Time 2 & 35 & 24.3 & 27.2 & 12 & 44.3 & 13.8 & 38 & 30.8 & 6.0 & 107 & 30.0 & 19.6 & 0.8 & $(-4.07 ; 5.71)$ & 0.3 & 163.3 & 0.741 \\
\hline $\begin{array}{l}\text { General estimate } \\
\text { equations }\end{array}$ & & & & & & & & & & & & & $\mathrm{B}$ (time) & $95 \% \mathrm{CI}$ & Wald Chi2 & $\mathrm{DF}$ & $\mathrm{p}$ \\
\hline \multicolumn{18}{|l|}{ Self-Esteem } \\
\hline Baseline - control & 46 & 25.0 & 0.0 & 18 & 29.2 & 5.5 & 49 & 25.1 & 1.7 & 85 & 25.7 & 2.9 & Ref & & & & \\
\hline Time 1 & 44 & 25.9 & 3.7 & 20 & 27.9 & 6.4 & 43 & 26.2 & 2.3 & 113 & 26.4 & 3.9 & 0.02 & $(-0.01 ; 0.06)$ & 2.1 & 1 & 0.149 \\
\hline Time 2 & 35 & 28.6 & 8.1 & 12 & 30.0 & 5.3 & 38 & 25.3 & 1.8 & 107 & 27.3 & 5.9 & 0.06 & $(0.02 ; 0.11)$ & 6.8 & 1 & 0.009 \\
\hline \multicolumn{18}{|l|}{ Disengagement } \\
\hline Baseline - control & 46 & 12.8 & 18.0 & 18 & 19.8 & 25.0 & 49 & 17.2 & 18.6 & 85 & 15.8 & 19.5 & Ref & & & & \\
\hline Time 1 & 44 & 14.3 & 20.3 & 20 & 19.0 & 24.0 & 43 & 13.6 & 14.1 & 113 & 14.9 & 18.8 & -0.12 & $(-0.44 ; 0.20)$ & 0.6 & 1 & 0.452 \\
\hline Time 2 & 35 & 12.0 & 19.2 & 12 & 15.9 & 16.0 & 38 & 21.0 & 18.3 & 107 & 16.6 & 18.7 & -0.05 & $(-0.38 ; 0.29)$ & 0.1 & 1 & 0.793 \\
\hline \multicolumn{18}{|l|}{ Sadness } \\
\hline Baseline - control & 46 & 1.7 & 10.0 & 18 & 2.1 & 6.4 & 49 & 1.6 & 6.0 & 85 & 1.7 & 7.8 & Ref & & & & \\
\hline Time 1 & 44 & 0.7 & 2.7 & 20 & 2.1 & 7.0 & 43 & 0.2 & 1.2 & 113 & 0.8 & 3.5 & -1.00 & $(-1.87 ;-0.13)$ & 5.1 & 1 & 0.024 \\
\hline Time 2 & 35 & 2.1 & 11.3 & 12 & 0.4 & 1.3 & 38 & 1.5 & 6.8 & 107 & 1.6 & 8.5 & -0.36 & $(-1.08 ; 0.36)$ & 1.0 & 1 & 0.323 \\
\hline \multicolumn{18}{|l|}{ Negative affect } \\
\hline Baseline - control & 46 & 2.9 & 11.4 & 18 & 2.8 & 5.0 & 49 & 1.6 & 4.5 & 85 & 2.3 & 8.1 & Ref & & & & \\
\hline Time 1 & 44 & 1.4 & 6.9 & 20 & 1.3 & 2.9 & 43 & 0.5 & 1.7 & 113 & 1.0 & 4.7 & -1.14 & $(-1.91 ;-0.37)$ & 8.4 & 1 & 0.004 \\
\hline Time 2 & 35 & 0.3 & 1.3 & 12 & 1.7 & 2.6 & 38 & 0.7 & 3.2 & 107 & 0.7 & 2.5 & -1.24 & $(-2.47 ;-0.01)$ & 3.9 & 1 & 0.049 \\
\hline
\end{tabular}

*The estimate represents the estimated difference of the scores over time, with the baseline score as the reference category.

Means are unadiusted.

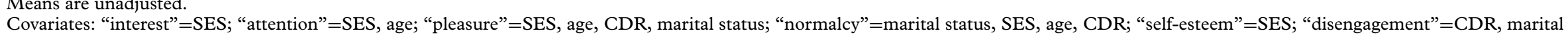
Covariates: "interest"=SES; "attention"=SES,
status, age, SES; "negative affect" $=$ SES, age. 
Table 3. Longitudinal changes for quality of life and communication

\begin{tabular}{|c|c|c|c|c|c|c|c|c|c|c|c|c|c|c|c|c|c|}
\hline & \multicolumn{3}{|c|}{$\begin{array}{c}\text { SITE } 1 \\
\text { CARE HOMES }\end{array}$} & \multicolumn{3}{|c|}{$\begin{array}{c}\text { SITE } 2 \\
\text { NHS UNITS }\end{array}$} & \multicolumn{3}{|c|}{$\begin{array}{c}\text { SITE } 3 \\
\text { COMMUNITY }\end{array}$} & \multicolumn{3}{|c|}{ TOTAL SAMPLE } & \multicolumn{5}{|c|}{ TIME } \\
\hline & $N$ & MEAN & $(\mathrm{SD})$ & $N$ & MEAN & $(\mathrm{SD})$ & $N$ & MEAN & $(\mathrm{SD})$ & $N$ & MEAN & $(\mathrm{SD})$ & ESTIMATE* & $95 \% \mathrm{CI}$ & $\mathrm{T}$ & $\mathrm{DF}$ & $\mathrm{p}$ \\
\hline \multicolumn{18}{|c|}{ Self-reported quality of life (DEMQOL, 28-112) } \\
\hline Baseline & 5 & 86.2 & 15.7 & 15 & 91.5 & 14.0 & 54 & 92.2 & 14.5 & 74 & 91.6 & 14.3 & & & & & \\
\hline Time $2-3$ months & 4 & 81.6 & 11.6 & 13 & 92.5 & 10.7 & 46 & 94.6 & 10.5 & 63 & 93.3 & 10.9 & 1.4 & $(-1.39 ; 4.23)$ & 1.0 & 103.8 & 0.32 \\
\hline Time $3-6$ months & 4 & 76.8 & 12.3 & 12 & 90.3 & 14.6 & 45 & 90.7 & 13.2 & 61 & 89.7 & 13.6 & -2.1 & $(-4.88 ; 0.77)$ & -1.4 & 103.9 & 0.15 \\
\hline \multicolumn{18}{|c|}{ Proxy rated quality of life (DEMQOL Proxy, $31-\underline{124}$ ) } \\
\hline Baseline & 48 & 98.9 & 16.6 & 19 & 86.7 & 12.6 & 43 & 86.3 & 15.2 & 110 & 91.9 & 16.5 & & & & & \\
\hline Time $2-3$ months & 41 & 100.1 & 12.3 & 9 & 96.3 & 10.2 & 44 & 91.2 & 14.8 & 94 & 95.6 & 13.9 & 3.1 & $(0.33 ; 5.78)$ & 2.2 & 159.4 & 0.03 \\
\hline Time $3-6$ months & 38 & 94.1 & 13.1 & 4 & 85.5 & 15.6 & 45 & 88.3 & 13.6 & 87 & 90.7 & 13.7 & -1.4 & $(-4.19 ; 1.43)$ & -1.0 & 161.3 & 0.33 \\
\hline \multicolumn{18}{|c|}{ Communication and social behavior (HOLDEN scale, $\underline{0}-48$ ) } \\
\hline Baseline & 48 & 20.6 & 10.8 & 19 & 12.9 & 9.5 & 54 & 13.1 & 7.4 & 121 & 16.0 & 9.9 & & & & & \\
\hline Time $2-3$ months & 41 & 18.5 & 11.1 & 15 & 19.3 & 10.0 & 44 & 13.0 & 7.7 & 100 & 16.2 & 9.9 & -0.1 & $(-1.37 ; 1.14)$ & -0.2 & 201.3 & 0.86 \\
\hline Time $3-6$ months & 38 & 19.8 & 12.8 & 9 & 20.7 & 10.9 & 46 & 14.9 & 8.1 & 93 & 17.5 & 10.7 & 1.5 & $(0.25 ; 2.83)$ & 2.4 & 202.2 & 0.02 \\
\hline
\end{tabular}

*The estimate represents the estimated difference of the scores over time, with the baseline score as the reference category.

Means are unadjusted.

Covariates: DEMQOL adjusted for SES; HCS adjusted for age, marital status and CDR score. 


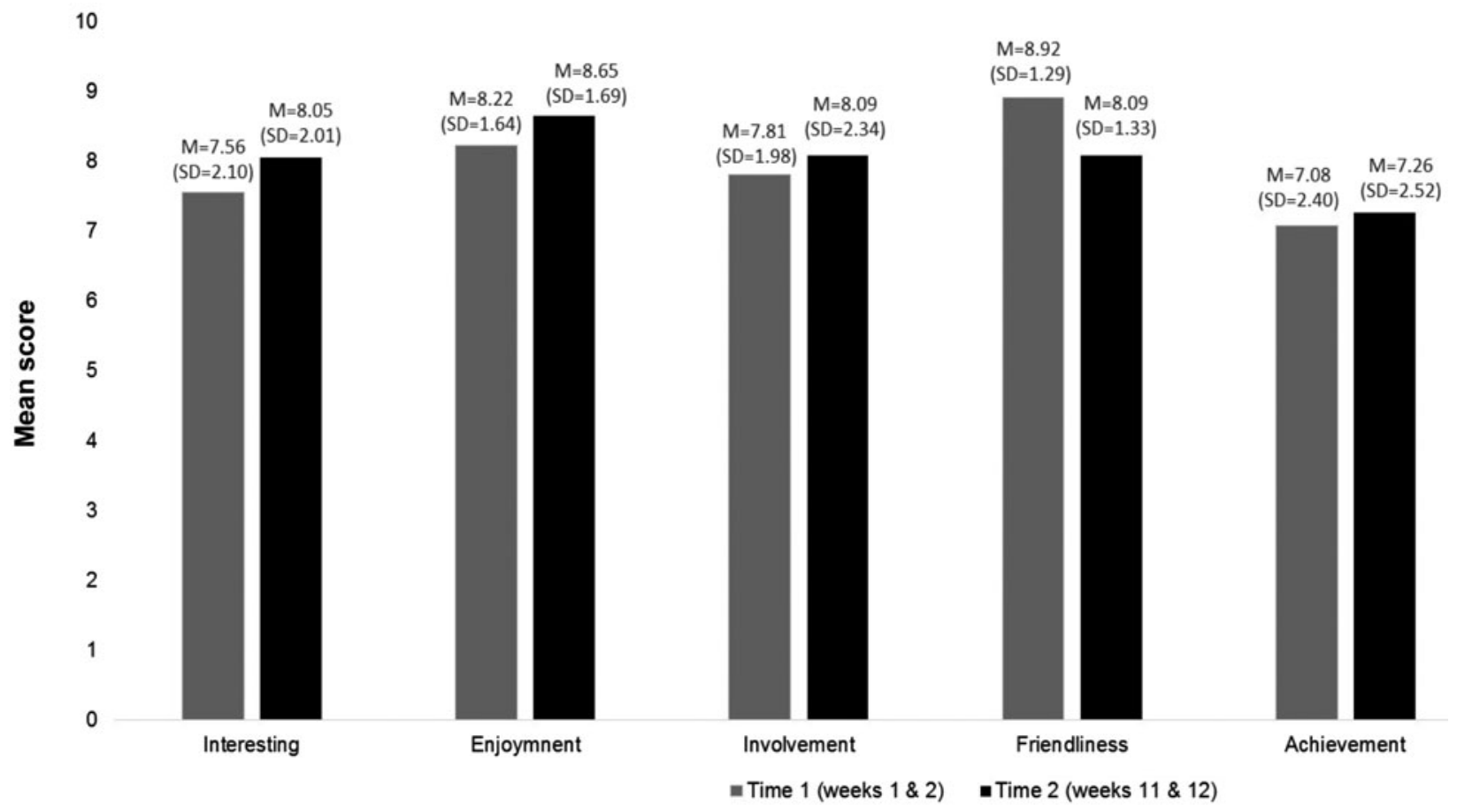

Figure 2. Descriptive statistics (mean scores) for session evaluations. The questions include the following: (1) How interesting was the session? (2) How much did you enjoy it? (3) How involved did you feel? (4) How friendly was the session? (5) Looking at what you have done today, how much do you feel you have achieved?

investigation to take this approach. The application of a mixed-methods research design to a much larger sample of participants and extended with a long-term follow-up adds to previous research of MacPherson et al. (2009); Camic et al. (2014). Across all the participants, when comparing the arts program with an active control, our findings suggest they experienced "in the moment" benefits to their well-being, in terms of improvements to "interest," "attention," "self-esteem," and "pleasure" and decreases in "negative affect" and "sadness." Ongoing weekly exposure to the art program may have been beneficial; "self-esteem" significantly increased by the end of the program at time 2. Similarly, across both intervention time-points "interest" significantly increased and "negative affect" significantly decreased compared to the control condition. Correspondingly, the immediate post-session evaluations (provided by participants at the same time-point as the observation data collection) were positively scored in relation to the intervention being interesting, friendly, and enjoyable. Participants reported a high sense of achievement and felt involved in the session.

Improvements in the DEMQOL-Proxy persisted beyond "the moment" from baseline (preintervention) to time 2 (at the end of the 12-week intervention), which were not sustained at time 3. In contrast, the self-rated DEMQOL from those who were able to complete did not show any significant differences over time. However, this was contrasted by qualitative reports (collected in the same interview) where the participants reported the intervention as a stimulating experience, noting a range of preferences and opinions on the role of the arts in relation to their condition, and the impact in terms of social connectedness, well-being and inner-strength.

On the whole, our results suggest the art program was equally beneficial regardless of the setting and the level of impairment. It builds on studies delivered in a gallery setting for people with mild to moderate dementia (e.g. Camic et al., 2014), and people with mild to severe dementia (MacPherson et al., 2009), and in a care-setting for people with mild to moderate dementia (Ullán et al., 2012). However, for the whole sample, scores on the HCS did not change from baseline to time 2, but worsened between baseline and time 3. The interaction by site found a deterioration in communication in the NHS participants across the study, but an improvement in the care home participants at time 2, although this was not sustained when the arts program was no longer being delivered. From this, we could infer the arts program may be particularly important for sustaining communication in a residential care environment. 
The environment is an important contextual consideration. While one aspect of dementia is changes in cognition, the other is how this influences, and is influenced by the social environment. Spector et al. (2003) also report variations in the HCS according to the setting following a cognitive stimulation intervention, suggesting some settings may be less able to generate good quality social environments, appearing more "institutionalized." This may be particularly pertinent to the NHS setting in this study, as many of the participants were there at a critical point of their condition, possibly with different, more intensive care needs than the participants in the other settings. Also, this NHS study site was unable to experience a gallery visit. Camic et al. (2015) discuss whether a gallery setting, considered a "valued place," is a different experience and so has a different impact compared to other settings. Our findings suggest the environment may be particularly relevant for communication and social behavior.

The lack of significant (or sustained) improvement at long-term follow-up (3 months postintervention) would suggest the weekly attendance may have made some difference to outcomes. Research with other psychosocial interventions indicates sustainability of effects is rare without further maintenance sessions (Orrell et al., 2014). Consequently, regular, and good quality activities should be a core component of care. Further research with a control-comparison group may help elucidate the extent to which benefits may occur beyond the activity.

\section{Strengths and weaknesses}

Reflecting guidance for developing and evaluating complex interventions (Medical Research Council, 2006), we sought to build on previous theory and research through a mixed-methods study design. The different modes of data collection each have their own strengths and weaknesses, but together are able to capture a more complete picture of impact. In terms of visual arts programs there has been little application to date of standardized, validated, and reliable QoL measures. Camic et al. (2014) included the DEMQOL, and while their qualitative data was informative, the quantitative data did not reveal any change, perhaps influenced by the small sample size $(n=28)$. With our larger sample, we found a significant improvement for the DEMQOL-Proxy but no significant effects for the DEMQOL. This is in contrast to suggestions that proxy reports of QoL are often under-estimated compared to patient self-assessment (Crocker et al., 2015).
Therefore, we concur with de Medeiros and Basting (2013) that where possible the subjective, individual experience should also be included in arts and dementia research, to augment or contrast quantitative findings. We suggest that qualitative approaches capture deeper meaning that could be over-looked by standardized measurement tools, and may be particularly useful for understanding the complexity of both the content of the arts program and the impact on concepts, such as QoL and well-being.

Standardized questionnaires or semi-structured interviews may however fail to capture what happens during program delivery. Inspired by MacPherson et al. (2009), we applied structured observation, exploring the extent to which aspects of well-being are influenced by the arts program "in the moment" and whether this is more beneficial than an alternative activity. Structured observation does not rely on the people living with dementia, their carers or indeed the researcher, to recall feelings and details, and enables a way of quantitatively capturing "in the moment" reactions that might otherwise be overlooked, or prove difficult to articulate.

Drawing on the recommendations of a review of observational measures in dementia care (Algar et al., 2014), we utilized and adapted an "observed well-being" tool, originally developed specifically to measure well-being in response to visual art program (Kinney and Rentz, 2005). Observation measures can initially require more researchers' training to ensure inter-rater reliability, are time consuming to administer, and generate a substantial amount of data. Fortunately, our study was able to address these issues, with training and periodic quality checks. Consequently, our mixedmethods design was able to measure the effects "in the moment" and longitudinally, and understand the subjective, individual experiences.

Participant attrition was low, and we had $80.8 \%$ complete outcome data for the quantitative measures. Although there were differences between the sites for some of the baseline demographic and clinical factors, we adopted a robust data analysis strategy toward their potential to confound the results, improving on the method proposed in the protocol (Windle et al., 2016). Consequently, we can be confident in our findings. However, we had to modify the protocol to facilitate recruitment and delivery in the NHS site. Despite assurances at the outset, the recruitment and retention of participants in that site was difficult, highlighting the challenges of undertaking research in busy acute settings. However, we did not have difficulty recruiting participants in the other sites, in fact we recruited more than our initial target, and were able 
to offer all participants an opportunity to be part of the research.

The study design, while rigorously applied, means we cannot draw definitive conclusions about effectiveness. However, we actively recruited people to a program specifically developed for the purpose of the research, across multiple research sites, and applied an eligibility criteria to clearly define the target population. For definitive conclusions of effectiveness, further research is required. This could incorporate randomization with a matched comparison/control condition. In terms of our research program, further work is analyzing the longitudinal impact on the family and professional carers of our participants living with dementia, and will maximize the mixed-methods framework by fully integrating the data.

\section{Practice implications}

Our research suggests that art and cultural organizations and community venues are ideally situated for delivering high quality, socially inclusive, and mentally stimulating activities. Currently in the United Kingdom the National Institute of Health and Clinical Excellence (NICE) recommend cognitive stimulation groups as a psychosocial treatment for people living with mild to moderate dementia. Cognitive stimulation may be a key aspect of the art program described here, as there is congruence between the "guiding principles" of cognitive stimulation, as described by Aguirre and colleagues (2011) and aspects of the arts program. A possible future implementation pathway could be to consider an arts program as a follow-on or perhaps an alternative to a conventional cognitive stimulation group. The practice guide of our arts program (Parkinson et al., 2017), and plans for ongoing training to be available beyond the end of the research will support further implementation.

Our qualitative findings also suggest some important barriers to participation, which would need to be considered in order to minimize inequity in access. It is worth noting that arts activities may need some careful marketing and service providers will likely need to address persistent cultural barriers around the arts to encourage people to take part. Nevertheless, $37 \%$ of our participants had not previously engaged with art, suggesting that many people are willing to try activities if they are available. There is also a question regarding the extent to which the findings might be replicated with different ethnic groups, as we were unable to offer participation to people who did not have understanding of English or Welsh. In their review of published research, Windle et al. (2017) note that few studies reported any detail about ethnicity.
In contrast to other visual arts studies, which aimed to recruit participant dyads (e.g. Camic et al., 2014), it was not compulsory for staff and carers to attend in this research, although some did. We based this decision on MacPherson et al. (2009), who found that the presence of carers, despite their best intentions, created an unnecessary dependency, and did not allow the people with dementia to fully participate and articulate their opinion. The presence of staff and carers on occasion required careful management by the artist-facilitators, and we would recommend that service providers understand how to manage such situations to maximize the potential for the person with dementia.

\section{Conclusion}

Dementia is so often the focus of a medical model of deficit, and societal representations of dementia are predominated by fear of what will be lost. In contrast, our research suggests an alternative portrayal, highlighting the potential for creative aging within dementia care. This reflects the global policy interest in the social inclusion of people living with dementia, and for communities to be dementia friendly (Lin and Lewis, 2015), where people can experience good quality activities without the stigma of their condition. Based on our findings, we encourage dementia care providers and arts and cultural services to work toward embedding art activities within routine care provision.

\section{Conflict of interest}

None.

\section{Description of authors' roles}

GW led the study, secured funding (with BW, $\mathrm{CP}$, and $\mathrm{AN}$ as co-applicants) and drafted this manuscript. KJ was responsible for the statistical design of the study, undertaking quantitative analysis, and critically revising this manuscript. PvdV provided an independent statistical advice and contributed comments to the manuscript. BW contributed to the study design and statistical design, provided clinical oversight, and critically revising this manuscript. TH undertook the qualitative analysis and contributed comments to the manuscript. CHJ led the quality control and supervised data collection for the observation measure, collected data in site 3 and contributed comments to the manuscript. CP contributed to 
the study design, supervised the data collection for site 2 and contributed comments to the manuscript. AN contributed to the study design, supervised the data collection for site 1 and contributed comments to the manuscript.

\section{Acknowledgments}

Dementia and Imagination was funded as "Dementia and imagination: connecting communities and developing well-being through socially engaged visual arts practice," Grant Ref: AH/K00333X/1, by the AHRC and ESRC as a part of the CrossCouncil Connected Communities Programme. (Principal Investigator G. Windle; Co-Investigators A. Newman, C. Parkinson, V. Burholt, M. Baber, V. Tischler, B. Hounsome, D. O'Brien, B. Woods, R. T. Edwards). Many thanks to B. Hounsome for his role as co-investigator in the first year.

Regional and national collaborating partners in Dementia and Imagination are the following: Age Watch, Alzheimer's Society, the Arts Council of Wales, Denbighshire County Council, Engage Cymru, Derbyshire Community Health Service NHS, Equal Arts, the BALTIC Centre for Contemporary Art, Tyne and Wear Archives and Museums and Nottingham Contemporary Ltd.

$\mathrm{KJ}$ undertook this work as part of a fellowship awarded through a joint partnership with the Alzheimer's Society and Alzheimer Nederland (grant number WE.15-2015-02). Thanks to Dr. Mike Bird for his clinical expertise and helpful methodology suggestions at the start of the project.

\section{Supplementary material}

To view supplementary material for this article, please visit https://doi.org/10.1017/ S1041610217002162

\section{References}

Aguirre, E., Spector, A., Streater, A., Hoe, J., Woods, B. and Orrell, M. (2011). Making a Difference, vol. 2. UK: Hawker Publications.

Algar, K. (2012). Lost in Art Too...? Evaluation of Art Sessions Provided by Denbighshire County Council. Available at: http://dsdc.bangor.ac.uk/documents/service-evaluations/ Evaluation \%20of\%20Lost\%20in\%20Art\% 20too_FINAL\%20(May\%202012).pdf; last accessed 3 March 2017.

Algar, K., Woods, B. and Windle, G. (2014). Measuring the quality of life and well-being of people with dementia: a review of observational measures. Dementia: The International fournal of Social Research and Practice, 15, 832-857. doi: 10.1177/1471301214540163.
Braun, V. and Clarke, V. (2006). Using thematic analysis in psychology. Qualitative Research in Psychology, 3, 77-101.

Camic, P., Baker, E. and Tischler, V. (2015). Theorizing how art gallery interventions impact on people with dementia and their caregivers. The Gerontologist, 56, 1033-1041. doi: 10.1093/geront/gnv063.

Camic, P. M., Tischler, V. and Pearman, C. H. (2014): Viewing and making art together: a multi-session art-gallery-based intervention for people with dementia and their carers. Aging $\mathcal{E}$ Mental Health. 18, 161-168. doi: 10.1080/13607863.2013.818101.

Crocker, T. F., Smith, J. K. and Skevington, S.M. (2015). Family and professionals underestimate quality of life across diverse cultures and health conditions: systematic review. Fournal of Clinical Epidemiology, 68, 584-595. doi: 10.1016/j.jclinepi.2014.12.007.

de Medeiros, K. and Basting, A. (2013). "Shall i compare thee to a dose of donepezil?": cultural arts interventions in dementia care research. The Gerontologist, 54, 344-353. doi: 10.1093/geront/gnt055.

Gordon-Nesbitt, R. (2015). Exploring the longitudinal relationship between arts engagement and health. Arts for Health, Manchester Metropolitan University. Available at: http://www.artsforhealth.org/research/

artsengagementandhealth/ArtsEngagementandHealth.pdf; last accessed 23 January 2017.

Gregory, S. and Windle, G. (2013). Lost in Art Too. An evaluation of a 10 week programme of art sessions provided by Denbigh County Council for people with dementia and their carers. Available at: http:/dsdc.bangor.ac.uk/documents/ service-evaluations/Lost $\% 20$ in $\% 20$ Art $\% 20$ Report $\% 20$ (May\%2030\%202013).pdf; last accessed 6 October 2016.

Holden, U. P. and Woods, R. T. (1995). Positive Approaches to Dementia Care, 3rd edn, revised. Edinburgh: Churchill Livingstone.

Hughes, C. P., Berg, L., Danziger, W. L., Coben, L. A. and Martin, R. L. (1982). A new clinical scale for the staging of dementia. The British fournal of Psychiatry, 140 566-572. doi: 10.1192/bjp.140.6.566.

Kinney, J. M. and Rentz, C. A. (2005). Observed well-being among individuals with dementia: memories in the making, an art program, versus other structured activity. American fournal of Alzheimer's Disease and Other Dementias, 20, 220-227.

Lin, S-Y. and Lewis, F. M. (2015). Dementia friendly, dementia capable and dementia positive: concepts to prepare for the future. The Gerontologist, 55, 237-244. doi:10.1093/geront/gnu122.

MacPherson, S., Bird, M., Anderson, K., Davis, T. and Blair, A. (2009). An art gallery access programme for people with dementia: "you do it for the moment". Aging and Mental Health, 13, 744-752.

McDermott, O., Crellin, N., Ridder, H. M. and Orrell, M. (2013). Music therapy in dementia: a narrative synthesis systematic review. International fournal of Geriatric Psychiatry, 28, 781-794. doi: 10.1002/gps.3895.

Medical Research Council. (2006). Developing and evaluating complex interventions: new guidance. Available at: https:/www.mrc.ac.uk/documents/pdf/complexinterventions-guidance/; last accessed 12 October 2016.

Mental Health Foundation. (2011). Evidence review of the impact of participatory arts on older people. Mental Health 
Foundation. Available at: http://www.baringfoundation. org.uk/EvidenceReview.pdf; last accessed 2 February 2016.

Orrell, M. et al. (2014). Maintenance cognitive stimulation therapy for dementia: single-blind, multi-centre, pragmatic randomised controlled trial. British Fournal of Psychiatry, 204, 454-461. doi: 10.1192/bjp.bp.113.137414.

Parkinson, C., Windle, G., Taylor, K. and The Dementia and Imagination Team. (2017). Research informed approaches to visual arts programmes. Available at: http://www.artsforhealth.org/resources/ dementia-and-imagination.pdf; last accessed 5 February 2017.

Rothman, K. J. and Greenland, S. (1998). Modern Epidemiology, 2nd edn, revised. USA: Lippincott-Raven.

Salisbury, K., Algar, K. and Windle, G. (2011). Do we know enough about the potential of art programmes to improve the quality of life and well-being of people with dementia? Fournal of Dementia Care, 19, 33-37.

Sauer, P. E., Fopma-Loy, J., Kinney, J. and Lokon, E. (2014). It makes me feel like myself: Person-centered versus traditional visual activities for people with dementia. Dementia: The International fournal of Social Research and Practice. doi: 10.1177/1471301214543958.

Smith, S. C. et al. (2007). Development of a new measure of health-related quality of life for people with dementia: DEMQOL. Psychological Medicine, 37, 737-746.
Spector, A. et al. (2003). Efficacy of an evidence-based cognitive stimulation therapy programme for people with dementia. British fournal of Psychiatry, 183, 248-254. doi: 10.1192/bjp.183.3.248.

Ullán, A. M. et al. (2012). Contributions of an artistic educational program for older people with early dementia: an exploratory qualitative study. Dementia: The International Fournal of Social Research and Practice. doi: $10.1177 / 1471301211430650$.

Windle, G. et al. (2016). Dementia and Imagination: a mixed-methods protocol for arts and science research. BMF Open, 6, doi: 10.1136/bmjopen-2016-011634.

Windle, G., Howson-Griffiths, T., Gregory, S., O'Brien, D., Newman, A. and Goulding, A. (2017). Exploring the theoretical foundations of visual art programmes for people living with dementia. Dementia: The International Fournal of Social Research and Practice. Epub ahead of print, September 15, 2017.

Young, R., Camic, P. M. and Tischler, V. (2015). The impact of community-based arts and health interventions on cognition in people with dementia: a systematic literature review. Aging and Mental Health. doi: 10.1080/13607863.2015.1011080.

Zeilig, H., Killick, J. and Fox, C. (2014). The participative arts for people living with a dementia: a critical review. International Fournal of Ageing and Later Life, 9, $7-34$. 